\title{
Preparation and Characterization of NiO Nanoparticles by Anodic Arc Plasma Method
}

\author{
Hongxia Qiao, ${ }^{1,2}$ Zhiqiang Wei, ${ }^{2,3}$ Hua Yang, ${ }^{3}$ Lin Zhu, ${ }^{3}$ and Xiaoyan Yan $^{3}$ \\ ${ }^{1}$ School of Civil Engineering, Lanzhou University of Technology, Lanzhou 730050, Gansu, China \\ ${ }^{2}$ State Key Laboratory of Advanced New Nonferrous Materials, Lanzhou University of Technology, \\ Lanzhou 730050, Gansu, China \\ ${ }^{3}$ School of Science, Lanzhou University of Technology, Lanzhou 730050, Gansu, China
}

Correspondence should be addressed to Zhiqiang Wei, zqwei@lut.cn

Received 15 November 2008; Accepted 12 December 2008

Recommended by Min Xu

$\mathrm{NiO}$ nanoparticles with average particle size of $25 \mathrm{~nm}$ were successfully prepared by anodic arc plasma method. The composition, morphology, crystal microstructure, specific surface area, infrared spectra, and particle size distribution of product were analyzed by using X-ray diffraction (XRD), transmission electron microscopy (TEM) and the corresponding selected area electron diffraction (SAED), Fourier transform infrared (FTIR) spectrum, and Brunauer-Emmett-Teller (BET) $\mathrm{N}_{2}$ adsorption. The experiment results show that the $\mathrm{NiO}$ nanoparticles are bcc structure with spherical shape and well dispersed, the particle size distribution ranging from 15 to $45 \mathrm{~nm}$ with the average particle size is about $25 \mathrm{~nm}$, and the specific surface area is $33 \mathrm{~m}^{2} / \mathrm{g}$. The infrared absorption band of $\mathrm{NiO}$ nanoparticles shows blue shifts compared with that of bulk NiO.

Copyright (C) 2009 Hongxia Qiao et al. This is an open access article distributed under the Creative Commons Attribution License, which permits unrestricted use, distribution, and reproduction in any medium, provided the original work is properly cited.

\section{Introduction}

Nanoparticles exhibit novel properties that significantly differ from those of corresponding bulk solid state owing to the different effects in terms of small size effect, surface effect, quantum size effect, and macroscopic quantum tunnel effect $[1,2]$. In recent years, $\mathrm{NiO}$ nanoparticles as a kind of functional material has attracted extensive interests due to its novel optical, electronic, magnetic, thermal, and mechanical properties and potential application in catalyst, battery electrodes, gas sensors, electrochemic films, photoelectronic devices, and so on [3-9]. In these applications, it is still needed for synthesizing high-quality and ultrafine powders with required characteristics in terms of their size, morphology, microstructure, composition purity, crystallizability, and so on, which are the most essential factors which eventually determine the microstructure and performance of the final products. Therefore, it is very important to control the powder properties during the preparation process.
There are many different methods reported for the synthesis of $\mathrm{NiO}$ nanoparticles, such as ultrasonic radiation, hydrothermal synthesis, carbonyl method, laser chemical method, pyrolysis by microwave, sol-gel method, precipitation-calcination, microemulsion method, and so on [10-15]. However, to the best of our knowledge, most of the reported experimental techniques for the synthesis of nanopowders are still limited in laboratory scale due to some unresolved problems, such as special conditions, tedious procedures, complex apparatus, low yield, and high cost. From a practical viewpoint, it is vital to develop a way to manufacture high-quality nanopowders at high throughput with low cost.

Anodic plasma method is an effective feasible process to prepare monodisperse metal nanopowders [16]. Compared with the conventional methods, anodic arc plasma method has many advantages: metal nanopowders prepared by this process with ultrafine particle size, higher purity, and narrow size distribution, well dispersed and spherical shape. Furthermore, the physical and chemical properties 
of the nanopowders can be easily controlled by varying the processing parameters and no need for expensive agents or special equipment. In addition, it is a convenient, inexpensive process, has high productive capacity and the potential for further mass production in the industry.

In this paper, $\mathrm{NiO}$ nanoparticles were successfully prepared by anodic arc plasma technique. In addition, the composition, morphology, microstructure, specific surface area, infrared spectra, the particle size, and distribution of product by this process were characterized via X-ray diffraction (XRD), transmission electron microscopy (TEM) and the corresponding selected area electron diffraction (SAED), Fourier transform infrared spectrum (FTIR), and BET $\mathrm{N}_{2}$ adsorption. The knowledge obtained would enhance a better understanding to the microstructure of $\mathrm{NiO}$ nanoparticles, and finally assist in the further development of novel properties and also benefit the practical industrial application in the future.

\section{Experimental}

The detailed experimental apparatus was fully illustrated elsewhere [16], it mainly includes the stainless steel vacuum chamber, the gas supply device, the DC power supply, the plasma generator with a high frequency initiator, the vacuum pump, the water-cooled collection cylinder, and the water-cooled copper crucible. The bulk pure material to be evaporated was laid on the water-cooled copper crucible, which served as the anode. Ni rod with $10 \mathrm{~mm}$ diameter is mounted in an insulated and axial manner, which was also water cooled and served as the cathode. In the process of preparation, the vacuum chamber was pumped to $10^{-3} \mathrm{~Pa}$ and then a mixture of $\mathrm{O}_{2}$ and $\operatorname{Ar}(1: 4$ by volume ratio) was backfilled as a reactant gas to reach the desired pressure. The electric arc in the inert environment was automatically ignited between the $\mathrm{Ni}$ electrode and the nozzle by highfrequency initiator, which was maintained by the current source at the preestablished values of the voltage and current. Under argon pressure and electric discharge current, the ionized gases were driven through the nozzle outlet and form the plasma jet. The bulk metal nickel (purity 99.99\%) was heated and melted by the high temperature, and metal atom detached from the metal surface when the plasma jet kinetic energy exceeds the metal superficial energy, and evaporated into atom soot. Above the evaporation source was a region of supersaturated metal vapor, where metal atoms diffused around and collided with $\mathrm{O}$ atom to form $\mathrm{NiO}$ at high temperature due to the oxidation reaction. When the vapor was supersaturated, a new phase was nucleated homogeneously out of the aerosol systems. The droplets were rapidly cooled and combined to form primary particles by an aggregation growth mechanism. The particles were transported from the nucleation and growth region to the inner walls of the cylinder by the free inert gas convection between the hot evaporation source and the cooled collection cylinder, the loose nanoparticles could be obtained.

The crystalline structures of the products were characterized by a rotating-target X-ray diffractometer (Japan
Rigaku D/Max-2400) equipped with a monochromatic highintensity $\mathrm{Cu}$ Ka radiation $(\lambda=1.54056 \AA, 40 \mathrm{kV}, 100 \mathrm{~mA})$. The sample was scanned in a range from 30 to $90^{\circ}(2 \theta)$ with scanning rate was $0.005^{\circ} / \mathrm{s}$ and step size $0.02^{\circ}$. The average crystalline grain size of the $\mathrm{NiO}$ nanoparticles was estimated from the half maximum width and the peak position of an XRD line broadened according the Scherrer formula, which was further confirmed by the transmission electron microscopy (TEM) results and BET results.

The particle size and morphology of the sample and the corresponding selected area electron diffraction (SAED) were examined by JEOL JEM-1200EX transmission electron microscopy (TEM) with an accelerating voltage of $80 \mathrm{kv}$. In the process of preparation of the TEM specimen, a small amount of the powders was dispersed in a few milliliters of ethanol in an ultrasonic bath and ultrasonic treated for 30 minutes, and a drop from an eye dropper of the resulting suspension was placed onto a carbon-coated copper grid. The samples were placed in a vacuum oven to dry at ambient temperature before examining. The sample is scanned in all zones before the picture is taken.

The specific surface area of the sample was calculated from the BET adsorption equation and measured by the accelerated surface area porosimetry (ASAP 2010) instrument produced by Micromerities Corp. at liquid nitrogen temperature $(78 \mathrm{~K})$.

The absorption spectroscopy spectra of the product were recorded from 400 to $4000 \mathrm{~cm}^{-1}$ on a Nicolet Nexus 670 Fourier transform infrared (FT-IR) spectrometer using the $\mathrm{KBr}$ pellet technique to determine the chemical structure of the sample

\section{Results and Discussion}

The purity and crystallinity of the as-synthesized $\mathrm{NiO}$ nanoparticles were examined by using powder X-ray diffraction (XRD) as shown in Figure 1. It can be seen from Figure 1 that the diffraction peaks are low and broad due to the small size effect and incomplete inner structure of the particle. The peaks positions appearing at $2 \theta=37.20^{\circ}$, $43.20,62.87^{\circ}, 75.20^{\circ}$, and $79.38^{\circ}$ can be readily indexed as (111), (200), (220), (311), and (222) crystal planes of the bulk NiO, respectively. All these diffraction peaks can be perfectly indexed to the face-centered cubic (FCC) crystalline structure of $\mathrm{NiO}$, not only in peak position, but also in their relative intensity of the characteristic peaks, which is in accordance with that of the standard spectrum (JCPDS, No. 04-0835). The XRD pattern shows that the samples are single phase and no any other impurities distinct diffraction peak except the characteristic peaks of FCC phase $\mathrm{NiO}$ was detected. This result shows that the physical phases of the $\mathrm{NiO}$ nanoparticles have higher purity prepared in this work. The NiO lattice constant calculated from the XRD data is $4.1729 \AA$, which is in good agreement with the reported data (JCPDS, No. 04-0835).

The average crystallite size is calculated by X-ray diffraction line broadening using the Scherrer formula $d=$ $K \lambda / B \cos \theta$, where $d$ represents the grain size; $K=0.89$ is 


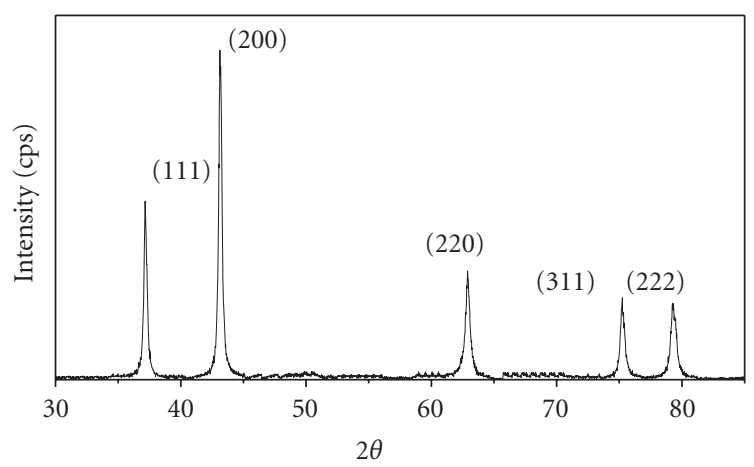

FIGURE 1: XRD pattern of NiO nanoparticles.

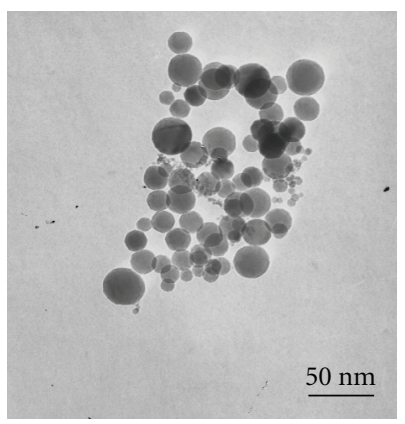

(a)

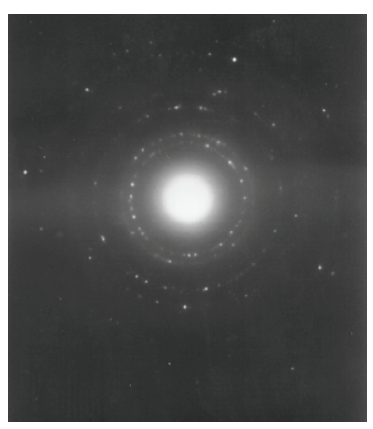

(b)
FIGURE 2: (a) TEM micrograph and (b) the selected area electron diffraction pattern of $\mathrm{NiO}$ nanoparticles.

the Scherrer constant related to the shape and index (hkl) of the crystals; $\lambda$ is the wavelength of the X-ray $(\mathrm{Cu} \mathrm{Ka}$, $1.54056 \AA$ ); $\theta$ is the diffraction angle of the peak; $B$ stands for the full width at half-height of the peaks (in radian) given by $B^{2}=B m^{2}-B s^{2}$, where $B m$ is the full width at half maximum (FWHM) of the sample and $B s$ is the half-width of a standard sample with a known crystal size greater than $100 \mathrm{~nm}$, the effect of instrumental broadening on the reflection peaks is calibrated. The crystallite sizes of $\mathrm{NiO}$ samples is $23 \mathrm{~nm}$ which was calculated from measured values for the spacing of the (111) plane.

Figure 2(a) shows the representative transmission electron microscopy image of $\mathrm{NiO}$ nanoparticles. TEM analysis of the products provided information on the size and morphology of $\mathrm{NiO}$ nanoparticles and their state of agglomeration. It can be seen from Figure 2(a) that the $\mathrm{NiO}$ nanoparticles had spherical shapes and were well dispersed with smooth surface and uniform size. Few small particles aggregate into secondary particles because of their extremely small dimensions and high surface energy.

Figure 2(b) shows the corresponding selected area electron diffraction (SAED) pattern. The SAED pattern consists of five diffraction rings with different radii and one center. The diameter of the diffraction ring in SAD pattern is proportional to $\sqrt{h^{2}+k^{2}+l^{2}}$, where $(\mathrm{hkl})$ are the Miller indices of the planes corresponding to the ring, counting

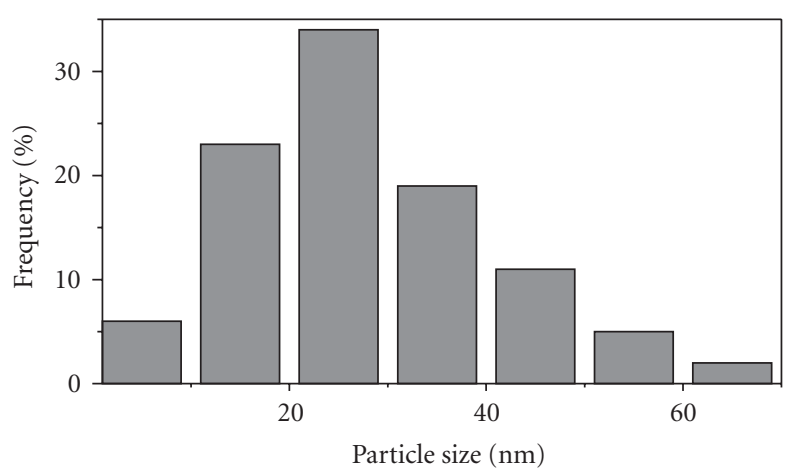

Figure 3: Particle size distribution of NiO nanoparticles.

from the center $1 \mathrm{st}, 2 \mathrm{nd}, 3 \mathrm{rd}, 4$ th, and 5 th rings correspond to (111), (200), (220), (311), and (222) planes, respectively. Tropism of the particles at random and small particles cause the widening of diffraction rings that made up of many diffraction spots, which indicates that the nanoparticles are polycrystalline structure. The SAED pattern also confirms that the $\mathrm{NiO}$ nanoparticles are face-centered cubic (FCC) structure $\mathrm{NiO}$ in crystallography, which consistent with the above X-ray results.

From the data obtained by TEM micrographs, the particle size histograms can be drawn and the mean size of the particles can be determined. Figure 3 shows the particle size distribution of $\mathrm{NiO}$ nanoparticles. It can be seen that the particle sizes possess a small and narrow size distribution in a range from 15 to $45 \mathrm{~nm}$, and the mean diameter (taken as average particle diameter) is about $25 \mathrm{~nm}$. We notice that the mean particle size determined by TEM is in good agreement with the average crystallite size calculated by the Scherer formula from the XRD patterns. According to the TEM image, it could be concluded that this preparation method had successfully overcome the problem of agglomeration and appropriate to obtain the $\mathrm{NiO}$ nanoparticles with smaller crystalline size.

The surface area analysis was carried out on $\mathrm{NiO}$ nanoparticles by BET method. Assuming the particles possess solid, spherical shape with smooth surface and same size, the surface area can be related to the average equivalent particle size by $D_{\mathrm{BET}}=6000 /\left(\rho \cdot S_{w}\right)$ (in nm), where $D_{\mathrm{BET}}$ is the average diameter of a spherical particle; $S_{w}$ represents the measured surface area of the powder in $\mathrm{m}^{2} / \mathrm{g} ; \rho$ is the theoretical density in $\mathrm{g} / \mathrm{cm}^{3}$. Figure 4 shows BET plots of $\mathrm{NiO}$ nanoparticles, the specific surface area of $\mathrm{NiO}$ nanoparticles calculated using the multipoint BET-equation is $33 \mathrm{~m}^{2} / \mathrm{g}$, and the calculated average equivalent particle size is $28 \mathrm{~nm}$. We noticed that the particle size obtained from the BET and the TEM methods agree very well with the result given by X-ray line broadening. The results of TEM observations and BET methods further confirmed and verified the relevant results obtained by XRD as mentioned above.

Figure 5 shows the FTIR spectra of $\mathrm{NiO}$ nanoparticles, which showed several significant absorption peaks. The broad absorption band in the region of $600-700 \mathrm{~cm}^{-1}$ is assigned to $\mathrm{Ni}-\mathrm{O}$ stretching vibration mode; the broadness 


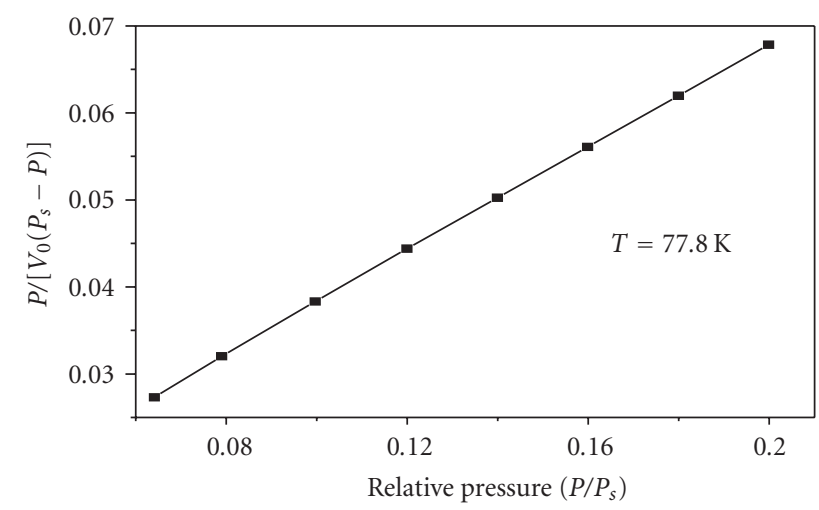

Figure 4: BET plots of $\mathrm{NiO}$ nanoparticles.

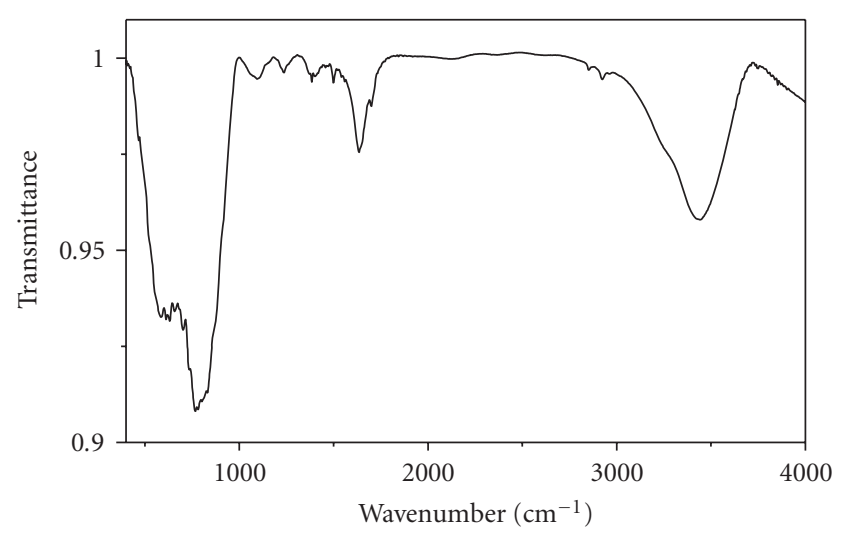

FIGURE 5: FTIR spectra of $\mathrm{NiO}$ nanoparticles.

of the absorption band indicates that the $\mathrm{NiO}$ powders are nanocrystals. The size of samples used in this study was much less than the bulks form $\mathrm{NiO}$, so that $\mathrm{NiO}$ nanoparticles had its IR peak of Ni-O stretching vibration and shifted to blue direction. Due to their quantum size effect and spherical nanostructures, the FTIR absorption of $\mathrm{NiO}$ nanoparticles is blue-shifted compared to that of the bulk form. Besides the $\mathrm{Ni}-\mathrm{O}$ vibration, it could be seen from Figure 5 that the broad absorption band centered at $3440 \mathrm{~cm}^{-1}$ is attributable to the band $\mathrm{O}-\mathrm{H}$ stretching vibrations and the weak band near $1635 \mathrm{~cm}^{-1}$ is assigned to $\mathrm{H}-\mathrm{O}-\mathrm{H}$ bending vibrations mode were also presented due to the adsorption of water in air when FTIR sample disks were prepared in an open air. These observations provided the evidence to the effect of hydration in the structure. Meanwhile, it implied the presence of hydroxyl in the precursor, and the broad absorption around $767 \mathrm{~cm}^{-1}$ is assigned to the band $\mathrm{C}=\mathrm{O}$ stretching vibrations. The serrated absorption bands in the region of 1000 $1500 \mathrm{~cm}^{-1}$ are assigned to the $\mathrm{O}-\mathrm{C}=\mathrm{O}$ symmetric and asymmetric stretching vibrations and the $\mathrm{C}-\mathrm{O}$ stretching vibration, but the intensity of the band has weakened, which indicated that the ultrafine powers tend to strong physically absorption to $\mathrm{H}_{2} \mathrm{O}$ and $\mathrm{CO}_{2}$.

\section{Conclusions}

(1) Cubic $\mathrm{NiO}$ nanoparticles with spherical shape higher purity, well dispersed, and narrow size distribution ranges from 15 to $65 \mathrm{~nm}$ were successfully prepared by anodic arc plasma method and different approaches such as XRD, TEM, SAED, FTIR, and BET were used to characterize.

(2) The specific surface area of the sample is $33 \mathrm{~m}^{2} / \mathrm{g}$ calculated from the BET adsorption equation, the average particle size about $25 \mathrm{~nm}$, the average equivalent particle size obtained from the TEM and confirmed by XRD and BET results. The infrared absorption band of the $\mathrm{NiO}$ nanoparticles show blue shifts compared with that of bulk $\mathrm{NiO}$.

\section{Acknowledgments}

This work was supported by the Key Project of Chinese Ministry of Education (no. 208151), Natural Science Foundation of Gansu Province, China (no. 2007GS04821), and Scientific Research Developmental Foundation of Lanzhou University of Technology (no. SB10200805).

\section{References}

[1] H. Gleiter, "Nanocrystalline materials," Progress in Materials Science, vol. 33, no. 4, pp. 223-315, 1990.

[2] Z. L. Wang, Y. Liu, and Z. Zhang, Handbook of Nanophase and Nanostructed Materials, Tsinghua University Press, Beijing, China, 2002.

[3] T. Fukui, S. Ohara, H. Okawa, T. Hotta, and M. Naito, "Properties of $\mathrm{NiO}$ cathode coated with lithiated $\mathrm{Co}$ and $\mathrm{Ni}$ solid solution oxide for MCFCs," Journal of Power Sources, vol. 86, no. 1-2, pp. 340-346, 2000.

[4] Y. Izaki, Y. Mugikura, T. Watanabe, M. Kawase, and J. R. Selman, "Direct observation of the oxidation nickel in molten carbonate," Journal of Power Sources, vol. 75, no. 2, pp. 236243, 1998.

[5] I. Hotovy, J. Huran, L. Spiess, S. Hascik, and V. Rehacek, "Preparation of nickel oxide thin films for gas sensors applications," Sensors and Actuators B, vol. 57, no. 1-3, pp. 147-152, 1999.

[6] H. Bi, S. Li, Y. Zhang, and Y. Du, "Ferromagnetic-like behavior of ultrafine $\mathrm{NiO}$ nanocrystallites," Journal of Magnetism and Magnetic Materials, vol. 277, no. 3, pp. 363-367, 2004.

[7] Y. Ichiyanagi, N. Wakabayashi, J. Yamazaki, et al., "Magnetic properties of NiO nanoparticles," Physica B, vol. 329-333, part 2, pp. 862-863, 2003.

[8] V. Biju and M. Abdul Khadar, "Fourier transform infrared spectroscopy study of nanostructured nickel oxide," Spectrochimica Acta Part A, vol. 59, no. 1, pp. 121-134, 2003.

[9] G. Wang, L. Zhang, and J. Mou, "Preparation and optical absorption of nanometer-sized $\mathrm{NiO}$ powder," Acta PhysicoChimica Sinica, vol. 13, no. 5, pp. 445-448, 1997.

[10] W. Yan and J.-J. Ke, "Preparation of nickel oxide particles by decomposition of basic nickel carbonate in microwave absorbing additive," Materials Research Bulletin, vol. 31, no. 1, pp. 51-61, 1996.

[11] S.-L. Che, K. Takada, K. Takashima, O. Sakurai, K. Shinozaki, and N. Mizutani, "Preparation of dense spherical Ni particles and hollow $\mathrm{NiO}$ particles by spray pyrolysis," Journal of Materials Science, vol. 34, no. 6, pp. 1313-1318, 1999. 
[12] J. L. Katz and P. F. Miquel, "Syntheses and applications of oxides and mixed oxides produced by a flame process," Nanostructured Materials, vol. 4, no. 5, pp. 551-557, 1994.

[13] Y. D. Li, C. W. Li, X. F. Duan, H. Zheng, L. Li, and Y. Qian, "Preparation of nanocrystalline $\mathrm{NiO}$ in mixed solvent," Journal of China University of Science and Technology, vol. 27, no. 3, pp. 346-349, 1997.

[14] W. Wang, Y. Liu, C. Xu, C. Zheng, and G. Wang, "Synthesis of $\mathrm{NiO}$ nanorods by a novel simple precursor thermal decomposition approach," Chemical Physics Letters, vol. 362, no. 1-2, pp. 119-122, 2002.

[15] L. Xing, X. Y. Deng, and Y. Jin, "Experimental study on synthesis of $\mathrm{NiO}$ nanoparticles," Scripta Materialia, vol. 47, no. 4, pp. 219-224, 2002.

[16] Z. Wei, T. Xia, L. Bai, J. Wang, Z. Wu, and P. Yan, "Efficient preparation for Ni nanopowders by anodic arc plasma," Materials Letters, vol. 60, no. 6, pp. 766-770, 2006. 

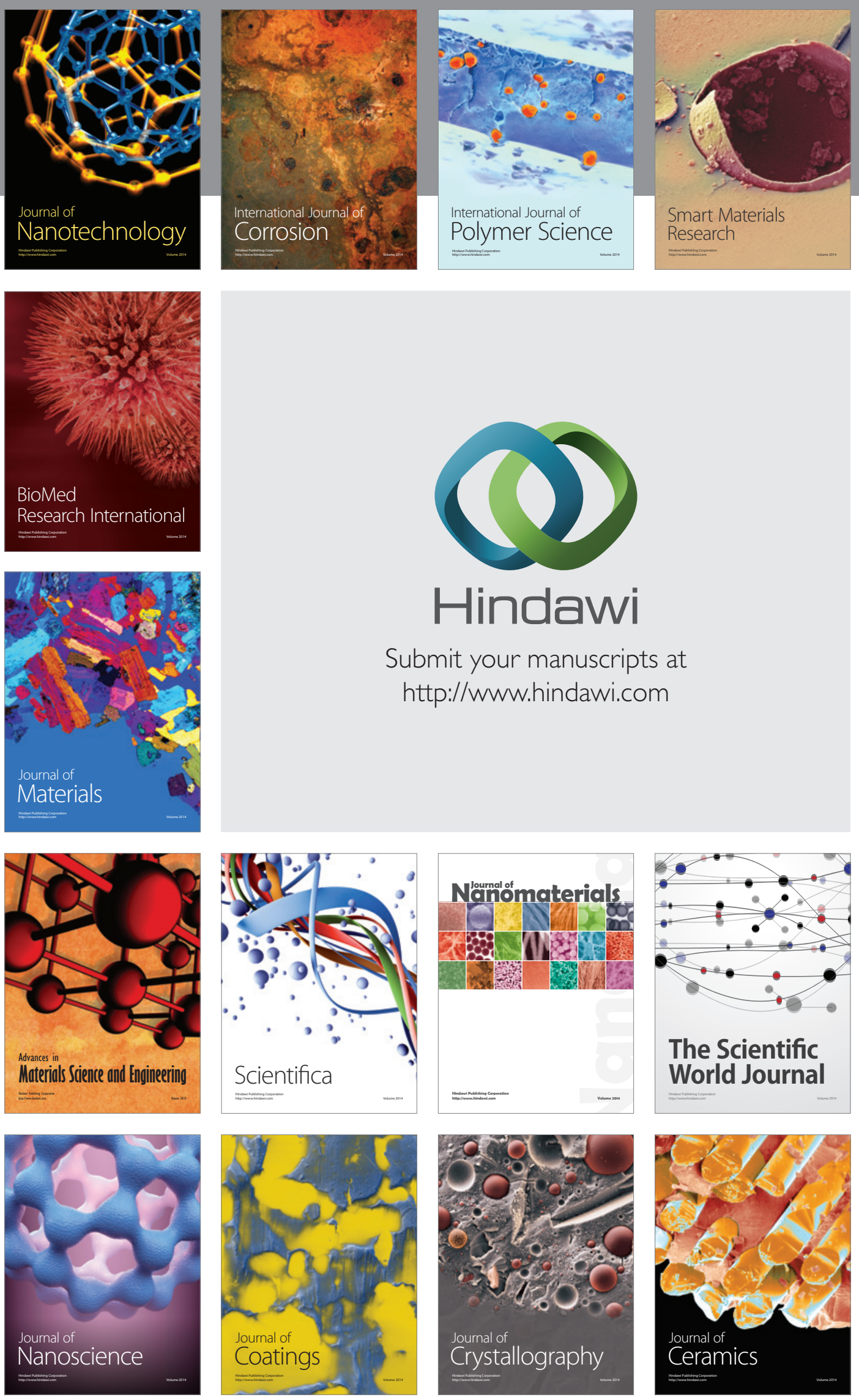

The Scientific World Journal

Submit your manuscripts at

http://www.hindawi.com

\section{World Journal}

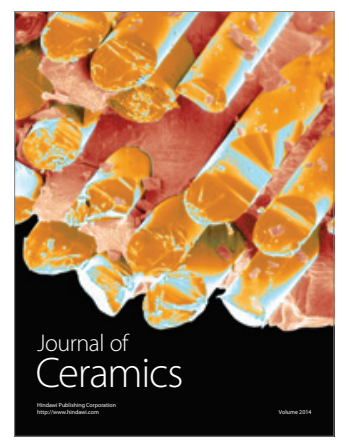

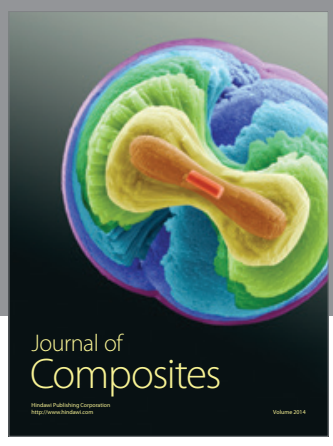
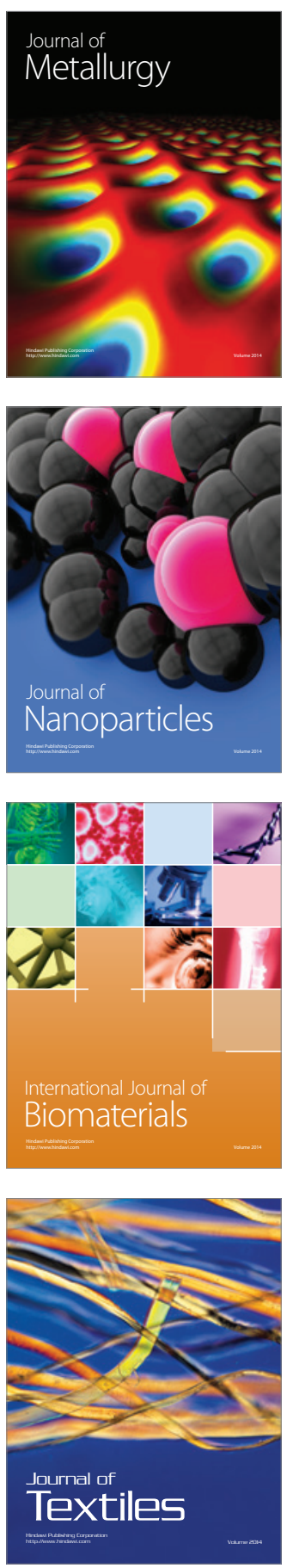